Hautarzt 2017 • 68:316-323

DOI 10.1007/s00105-017-3933-4

Online publiziert: 23. Januar 2017

(c) Der/die Autor(en) 2017. Dieser Artikel ist

eine Open-Access-Publikation.

CrossMark

\section{P. Mayser}

Biebertal, Deutschland

\section{Dermatophytid}

\section{Aktuelle Bestandsaufnahme}

Eine Id-Reaktion (-id [gr.]: Vater-SohnBeziehung) bezeichnet eine sekundäre entzündliche Reaktion auf ein entferntes umschriebenes immunologisches Geschehen. Im engeren Sinne kennzeichnet ein -id eine sterile Fernreaktion auf ein mikrobielles Allergen [1].

Jadassohn [2] prägte diesen Begriff 1918 anhand des von ihm beschriebenen Lichen trichophyticus, einer generalisierten feinpapulösen Reaktion auf eine ortsferne Dermatophytose (meist Kerion Celsi oder Tinea barbae).

Im Jahr 1928 schrieb Bloch: „Mit dem Namen Trichophytide bezeichnen wir in Analogie zu den, Tuberkuliden - Pilzerkrankungen, welche im Gefolge einer primären, exogenen, tiefen Trichophytie auf hämatogenem Weg in der allergischen Haut zustande kommen." Er unterschied Exanthemformen wie das lichenoide Trichophytid (Lichen trichophyticus) von makulopapulösen sowie polymorph-exsudativen und subkutannodösen Formen (Erythema nodosum trichophyticum). Ferner beobachtete er, dass eine Id-Reaktion vornehmlich auf der Höhe der Infektion bzw. bei hoher Allergenfreisetzung zu beobachten ist. Hierzu zählten damals die Röntgentherapie der Tinea capitis, aber auch der Trichophytin-Test sowie eine lokale Irritation [3].

Id-Reaktionen werden sehr häufig durch Pilzinfektionen (Mykide), insbesondere die Tinea pedis ausgelöst. Aber auch andere entzündliche oberflächliche Tineaformen, die Kandidose sowie subkutane (Sporotrichose) und tiefe Mykosen (Kokzidioidomykose) können IdReaktionen auslösen.

Der folgende Beitrag möchte einen aktuellen Überblick über das Krankheitskonzept, die pathogenetischen Hintergründe und die Bedeutung in der Praxis geben. Er wird sich dabei auf Dermatophyten-bedingte Mykide (enthalten in ICD-10: L 30.2) beschränken, da diese bezogen auf die Häufigkeit in der Praxis - die größte Rolle spielen.

\section{Id-Reaktionen bei Dermato- phyten-bedingten Mykosen}

Als Kriterien für ein Dermatophytid gelten:

- nachgewiesene Dermatophytose an einer anderen Lokalisation,

- ein Nachweis von Pilzelementen in den id-bedingten Effloreszenzen (Nativpräparat, Histopathologie negativ; Kultur: kein Wachstum eines Dermatophyten),

- oft zunächst Verschlechterung unter Therapie mit den heute verfügbaren, hochwirksamen systemischen Antimykotika,

- Abheilung nach Therapie der Dermatophytose [1].

In einer Pubmed-basierten Recherche (Abfrage 12/2016) fanden sich unter den Suchbegriffen Dermatophytid (37), Trichophytid (4), Microsporid (1), Mykid (0), mycid (0) insgesamt 42 Publikationen aus dem Zeitraum von 1932 bis heute, davon 13 aus dem Jahr 2000 und jünger. Diese geringe Zahl an Publikationen mag darauf zurückzuführen sein, dass Mykide in der Praxis nur noch selten diagnostiziert werden $[4,5]$. Ursächlich wird angegeben, dass die Therapie von Pilzinfektionen heute so frühzeitig erfolgt, dass profunde Pilzerkrankungen und damit die Voraussetzungen für eine Sensibilisierung nur noch selten auftreten [4]. Auf der anderen Seite steigen die Prävalenzen von Dermatophytenbedingten Infektionen wie Tinea pedis, Tinea capitis und Tinea unguium an $[6$,
7]. Aktuelle Zahlen, wie häufig Dermatophytide in der Praxis auftreten, sind aber nicht verfügbar.

Dermatophytide präsentieren sich vornehmlich als

- umschriebene vesikulöse Veränderungen im Bereich der Hände/Finger (dyshidrosiformes Mykid),

- symmetrische disseminierte kleinpapulöse oder papulovesikulöse ekzemartige lichenoide Reaktionen mit follikulärer Akzentuierung meist am Stamm (Lichen trichophyticus), oft bei Tinea capitis,

- Erythema nodosum bei tiefen Dermatophytosen [8].

Das klinische Bild des Mykids ist jedoch sehr variabel [1, 9-22]. Klinische Manifestationen umfassen auch eine Erysipel-ähnliche Dermatitis, psoriasiforme Veränderungen, das Erythema multiforme, das Erythema anulare centrifugum und urtikarielle Veränderungen. Mykide können grundsätzlich am Rumpf, an den Extremitäten und im Gesicht auftreten. Auch generalisierte Reaktionen sind möglich: Gianni et al. [11] beschrieben ein psoriasiformes Dermatophytid bei einem 11-jährigen Mädchen unter einer Microsporum-canis-Infektion.

\section{Dyshidrosiformes Mykid bei Tinea pedis}

Ein symmetrisches vesikulöses Mykid der Hände kann in Zusammenhang mit einer meist akut inflammatorischen Fußmykose auftreten [8, 12, 13]. Betroffen sind die Fingerzwischenräume, Fingerrücken, Handinnenflächen und gelegentlich auch die Handrücken, ausgeprägte Blasenbildung kann das Krankheitsbild komplizieren (Pompholyx-Typ). Eine 
Hier steht eine Anzeige.

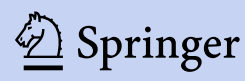




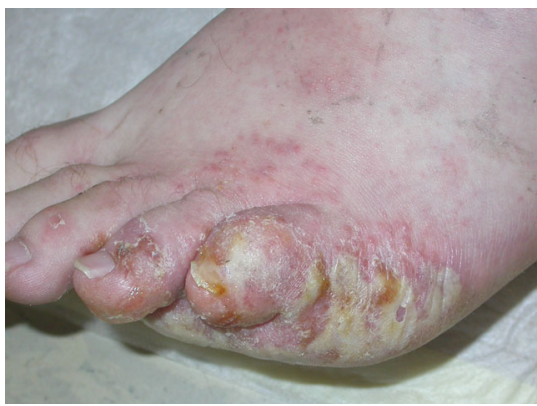

Abb. 1 A Eingetrübte Blasen im Bereich der linken Fußaußenseite

pustulöse Eintrübung der Bläschen wird bei Superinfektion beobachtet.

Das sterile dyshidrosiforme Mykid im Bereich der Hände ist von der eigentlichen Mykose im Bereich der Füße abzugrenzen, welches erschwert sein kann, wenn es sich um die vesikulösdyshidrotische Form der Tinea pedis handelt. Die Morphologie im Bereich der Füße bzw. der Hände kann sich dann identisch manifestieren, ebenso besteht im Bereich beider Lokalisationen heftiger Juckreiz. Die Tinea pedis ist jedoch oft einseitig ausgeprägt, während die hyperergen Veränderungen an den Händen weitgehend symmetrisch auftreten. Bei fehlender Schuppung gelingt der Pilznachweis aus dem Blasendach (Nativpräparat, Histologie) der vesikulösdyshidrotischen Tinea im Bereich der Füße, während sich aus den Veränderungen im Bereich der Hände keine Pilzelemente nachweisen lassen. Auslöser sind meist Trichophyton (T.) interdigitale und T. mentagrophytes.

\section{》) Es besteht heftiger Juckreiz}

Ein Fallbeispiel zeigt die klinischen Besonderheiten des dyshidrosiformen Mykids bei einer Tinea pedis. Ein 19jähriger Patient bemerkte 4 Wochen vor Erstvorstellung Juckreiz und Bläschenbildung zunächst am linken Fuß, 2 Wochen später auch im Bereich beider Hände. Bisher wendete er ein Ciclopiroxolamin-haltiges Gel an, sonst sind keine weiteren Erkrankungen bekannt. Es fanden sich im Bereich des linken Fußaußenrandes unscharf begrenzte Papeln, die zentral zu größeren

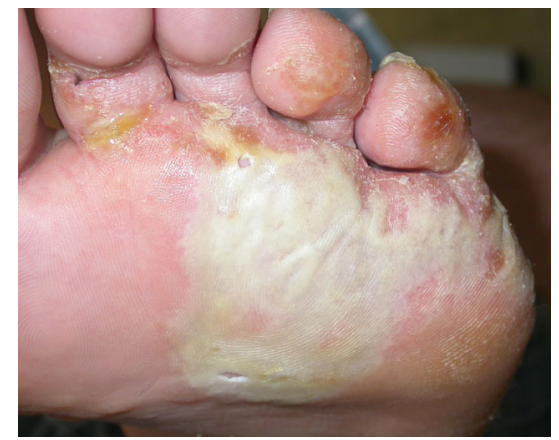

Abb. 2 ム Großblasige Ablösung der Fußsohle, interdigitale Mazeration

Blasen mit eitrig-putridem Inhalt konfluierten (• Abb. 1). Ferner zeigten sich eine Mazeration im Bereich des 3. und 4. Zehenzwischenraumes sowie Bläschen im Bereich der Zehen (• Abb. 2). Im Bereich beider Hohlhände sowie der Fingerzwischenräume war die Blasenbildung nahezu symmetrisch ausgeprägt (- Abb. 3a und b). Laborchemisch zeigte sich ein mäßig erhöhtes C-reaktives Protein (20,1 mg/l). Der Abstrich vom linken Fußaußenrand erbrachte massenhaft Staphylococcus aureus. Ein Stück Blasendecke aus dem Bereich des linken Vorfußes ergab eine kompakte Orthohyperkeratose mit eingebetteten PASpositiven Hyphen (• Abb. 4). Das klassische Kalilauge $(\mathrm{KOH})$-Nativpräparat von Schuppenmaterial aus dem Bereich des Fußaußenrandes war positiv, in der Kultur auf Selektivagar für pathogene Pilze (Merck, Darmstadt, FRG) nach 7 Tagen Wachstum von T. mentagrophytes. Schuppen aus dem Bereich der Hände waren nativmikroskopisch negativ, in der Kultur kein Nachweis eines Dermatophyten. Nach Diagnosestellung eines dyshidrosiformen Mykids bei Tinea pedis erfolgte eine Therapie mit Terbinafin $250 \mathrm{mg} / \mathrm{Tag}$ über 2 Wochen, zudem Prednisolon $50 \mathrm{mg}$ oral über 2 Tage mit einer Reduktion auf $25 \mathrm{mg}$ über weitere 2 Tage. Zusätzlich wurde topisch eine Kombination aus einem mittelstark wirksamen Kortikosteroid (Flupredniden-21-acetat) mit einem Breitspektrumantimykotikum (Miconazol) zur 2-maligen täglichen Anwendung verordnet. Schon nach 3 Tagen waren die entzündlichen Veränderungen deutlich rückläufig (• Abb.5) Die topische Kombinationstherapie wurde noch 10 Tage fortgesetzt, im Bereich der Hände wurde Mometason, ein topisches Steroid der Klasse III, in einer Cremegrundlage über ebenfalls 10 Tage verwendet.

Das dyshidrosiforme Mykid bei Tinea pedis ist keine seltene Erkrankung. Unter den 212 Patienten mit Fußmykose, die von Veien et al. [14] über 2 Jahre untersucht wurden, entwickelten $37 \mathrm{~Pa}$ tienten (17\%) ein Mykid, und bei 34 dieser Patienten waren ausschließlich die Hände betroffen. Bei 78 Patienten wurde die Infektion durch T. mentagrophytes verursacht. Von diesen entwickelten 27 (35\%) ein Mykid, während dies bei den 128 Patienten mit einer Infektion durch T. rubrum nur in 9 Fällen (7\%) auftrat. Bei einem der 6 mit Epidermophyton floccosum infizierten Patienten fand sich ein Mykid (16,67\%).

Diese Beobachtungen stimmen mit denen von Kaaman [15] überein, der anhand von Trichophytin-Testungen zeigte, dass T. mentagrophytes häufiger Immunreaktionen vom Spättyp induzierte als T. rubrum.

Inwieweit die durch die Ausbildung eines Mykids angezeigte starke Immunreaktion vom verzögerten Typ zu einer vollständigen Abheilung der Mykose führen kann, ist insbesondere bei den Mykosen im Bereich der Leistenhaut unklar [12-15]. Reservoire des Erregers könnten hier in der ausgeprägten Hornschicht verbleiben, sodass ein rezidivierender schubweiser Verlauf resultieren kann, wie er bei dyshidrosiformen Tineaformen nicht selten beobachtet wird.

\section{Dermatophytide vornehmlich bei Tinea capitis - Lichen trichophyticus und Erythema nodosum}

Bei dem Lichen trichophyticus handelt es sich um symmetrisch am Stamm gruppiert stehende, blasse, erythematöse, spitzkegelige, follikuläre Papeln. Bei ausgeprägten Verläufen können auch Gesicht und Extremitäten einbezogen sein. Ein Hinweis ist das gleichzeitige Bestehen einer oft massiven Dermatophytose [8].

Dostrovsky et al. [16] berichteten, dass innerhalb des Beobachtungszeitraumes 
von 1926 bis 1953 nur $0,2 \%$ von 6390 an einer Tinea capitis Erkrankten ein Mykid entwickelten. Die Id-Reaktionen standen üblicherweise mit einer therapeutischen Röntgentherapie in Verbindung, systemische Antimykotika wie Griseofulvin waren zu dieser Zeit noch nicht verfügbar. Grappel et al. [9] geben in einer $\mathrm{Pu}$ blikation aus dem Jahr 1974 eine Dermatophytidinzidenz bei Tineapatienten von $4,2 \%$ bei Kindern (vornehmlich Tinea capitis) und $4,6 \%$ bei Erwachsenen (vornehmlich Tinea pedis) an.

Cheng et al. [17] vermuteten 2011, dass ein Dermatophytid bei einer Tinea capitis häufiger auftritt als angenommen und wahrscheinlich öfter als Arzneimittelreaktion auf die antimykotische Therapie angesehen wird. Fünf Kinder (4 bis 7 Jahre; 2 Jungen, 3 Mädchen) mit einer Tinea capitis durch T. tonsurans entwickelten vor $(n=1)$ bzw. unter Therapie mit Griseofulvin $(n=4)$ kleinpapulöse, ekzematöse Veränderungen im Bereich des Kopfes bzw. Körperstammes mit Juckreiz. Die Veränderungen heilten unter Fortsetzen der systemischen antimykotischen Therapie ab.

In einer prospektiven Studie von Topaloğlu et al. [18] wurden kontinuierlich über etwa 8 Monate alle Kinder mit einem Kerion Celsi (6 Mädchen, 13 Jungen) auf das Vorhandensein eines Dermatophytids untersucht. Es manifestierte sich bei $68 \%$ (13/19), und zwar als Ekzemreaktion (7), kleine isolierte Papeln (3) oder eine Kombination von beiden (2). Ein Patient entwickelte Angioödemähnliche Symptome. Alle Dermatophytide bestanden vor Therapieeinleitung.

Auch über pustulöse Veränderungen wird vermehrt berichtet.

In dem Bericht von Liu [19] fanden sich bei einem 8-jährigen Mädchen mit Kerion Celsi durch T. mentagrophytes ein sich von Gesicht und Nacken rasch ausbreitendes, sehr stark juckendes Erythem mit kleinen Pusteln sowie Fieber von $38,9^{\circ} \mathrm{C}$. Unter einer Therapie mit Itraconazol ( $5 \mathrm{mg} / \mathrm{kg}$ Körpergewicht [KG]/Tag für 4 Wochen) und Dexamethason $(0,075 \mathrm{mg} / \mathrm{kg} \mathrm{KG} /$ Tag für 1 Woche) heilte das Exanthem nach 3 Tagen ab.

Ronjat et al. [20] berichteten über 3 Kinder (2 Jungen: 6 bzw. 11 Jahre,

Hautarzt 2017-68:316-323 DOI 10.1007/s00105-017-3933-4

(c) Der/die Autor(en) 2017. Dieser Artikel ist eine Open-Access-Publikation.

\section{P. Mayser}

\section{Dermatophytid. Aktuelle Bestandsaufnahme}

\section{Zusammenfassung}

Nach aktueller Definition versteht man unter einem Mykid eine hypererge Reaktionslage, bei der auch fern vom Herd der Infektion Hauterscheinungen auftreten, in denen keine Pilze nachweisbar sind. Als Kriterien für ein Dermatophyten-bedingtes Mykid (Dermatophytid) gelten: 1. nachgewiesene Dermatophytose an einer anderen Lokalisation, 2. kein Nachweis von Pilzelementen in den id-bedingten Effloreszenzen, 3. oft zunächst Verschlechterung unter Therapie mit hochwirksamen systemischen Antimykotika, 4. Abheilung nach Therapie der Dermatophytose. Häufigstes Dermatophytid ist ein symmetrisches dyshidrosiformes Mykid der Hände in Zusammenhang mit einer meist akut inflammatorischen Fußmykose insbesondere durch Trichophyton mentagrophytes. Bei Tinea capitis können Dermatophytide neben dem Lichen trichophyticus eine große klinische Varianz aufweisen und treten nicht selten erst unter der erforderlichen systemischen antimykotischen Therapie auf. Sie müssen von einer Arzneireaktion abgegrenzt werden. Zusätzlich zu einer systemischen antimykotischen Therapie führt, ggf. neben einer kurzfristigen oralen Gabe von Steroiden, die topische Applikation einer Kombination aus einem Antimykotikum mit einem Glukokortikoid zu einem raschen Rückgang von Entzündung und Juckreiz, gerade wenn es nach Einleiten der systemischen antimykotischen Therapie zu einem Aufflammen der Entzündung kommt (Overtreatment-Phänomen).

Schlüsselwörter

Mykid - Lichen trichophyticus · OvertreatmentPhänomen · Antimykotika · Steroide

\section{Dermatophyte. Current situation}

\section{Abstract}

Currently, a mycid is defined as hyperergic reaction that develops from a remote localized infection and in which no fungus is detectable. Criteria for a mycid caused by a dermatophyte (dermatophytid) are (1) proven dermatophytosis elsewhere, (2) no evidence for fungal elements in the lesions of the -id reaction, (3) initially often worsening of the lesions under therapy with highly potent systemic antimycotics, and (4) clearing after the dermatophytosis has been treated sufficiently. The most common dermatophytid is a symmetrical dyshidrotic mycid of the hands in connection with an often highly inflammatory mycotic infection of the feet especially by Trichophyton mentagrophytes. In addition to lichen trichophyticus, dermatophytids associated with tinea capitis may show high clinical variability. They often occur under systemic antimycotic therapy and have to be differentiated from drug reactions. In addition to systemic antimycotic therapy and, if necessary, a short-term application of oral glucocorticoidsteroids, a topical combination of an antifungal and a glucocorticosteroid is effective. It leads to an immediate reduction of inflammation and pruritus, especially if initiation of systemic antifungal therapy has resulted in a flare-up reaction (overtreatment phenomenon).

Keywords

Mycid · Lichen trichophyticus · Overtreatment phenomenon · Antifungals · Steroids
1 Mädchen: 6 Jahre) mit einem Kerion Celsi (Erreger T. tonsurans, T. soudanense, T. mentagrophytes), die 2 bis 3 Tage nach Beginn einer Griseofulvin-Therapie (19-23 mg/kg/Tag) ein sich vom Kopf auf den Stamm ausbreitendes pustulöses Exanthem, begleitet in einem Fall von Fieber und einer entzündlichen Chondritis, entwickelten. Der Pustelabstrich war steril, und laborchemisch konnte keine Entzündung nachgewiesen werden.
Unter Fortführen der Griseofulvin-Therapie besserten sich die Veränderungen unter oraler bzw. topischer Steroidgabe. In Abgrenzung zu einer akuten generalisierten exanthematischen Pustulose (AGEP) wurde ein generalisiertes exanthematisches pustulöses Dermatophytid diagnostiziert.

Obwohl ein Erythema nodosum (EN) als Folge einer Tinea capitis in der neueren Literatur bisher selten beschrieben 


\section{Übersichten}
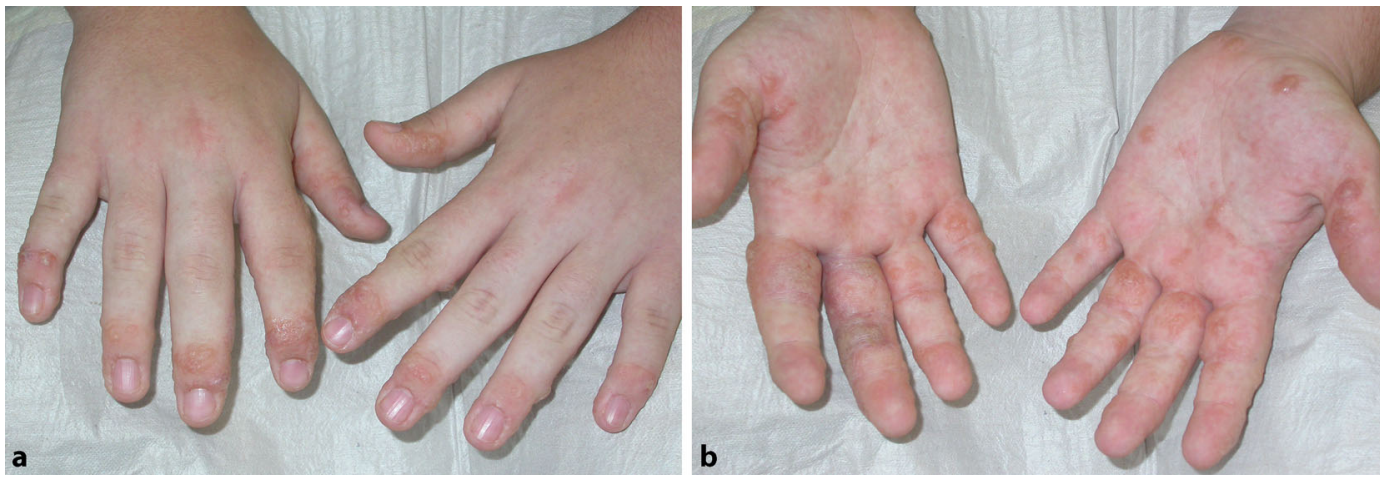

Abb. $3<$ a, b Massive Blasenbildung im Bereich der Hände, Pilznachweis negativ

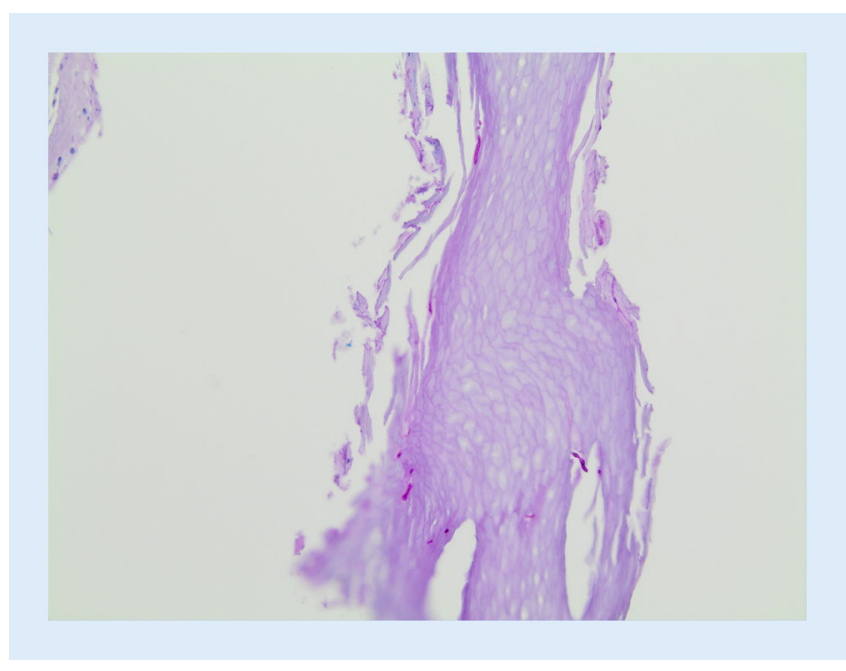

Abb. $4<$ Blasendecke mit Hyphe (PAS-Färbung, Vergr. 200:1) wurde, finden sich 2 aktuelle Fallberichte. Zaraa et al. [21] beschreiben das Auftreten eines EN bei einem 7-jährigen Jungen 2 Wochen nach Beginn eines Kerion durch T. mentagrophytes. Das histologisch gesicherte EN besserte sich 7 Tage nach Beginn einer Griseofulvin-Therapie.

Castriota et al. [22] berichteten über ein 9 Jahre altes Mädchen mit einem Kerion Celsi durch T. mentagrophytes, das nach Beginn einer 2-wöchigen Therapie mit Griseofulvin $25 \mathrm{mg} / \mathrm{kg} \mathrm{KG/Tag}$ (insgesamt 6 Wochen) ein EN entwickelte. Es folgte eine zusätzliche Gabe von Prednison $1 \mathrm{mg} / \mathrm{kg}$ KG/Tag über 10 Tage, worunter sich das EN zurückbildete. In ihrer Literaturübersicht stellen sie 14 weitere Fälle aus den Jahren 1945 bis 2013 zusammen, von denen 11 durch T. mentagrophytes verursacht wurden. In $7 \mathrm{der}$ insgesamt 15 Fälle trat das EN vor Beginn der systemischen antimykotischen Therapie auf.

\section{Diagnostik}

Bei einer vermuteten Dermatophytidreaktion ist die Suche nach bzw. der Nachweis einer Tinea mittels mykologischer Diagnostik (Nativpräparat, Kultur, Polymerasekettenreaktion [PCR]) entscheidend [8]. Oft handelt es sich um entzündliche Tineaformen mit stärkerer Entzündungsreaktion. In der differenzialdiagnostischen Abklärung ist insbesondere die Durchführung des Nativpräparates eine rasche und einfache Methode. Gerade bei dyshidrosiformen Veränderungen kann durch mehrere Probenentnahmen auch der Ort der eigentlichen Infektion eingegrenzt werden. Da aufgrund der Dicke der Hornschicht die Aussagekraft des KOHNativpräparates eingeschränkt sein kann (lange Mazerationszeit), kann alternativ oder ergänzend auch eine histologische Untersuchung (PAS-Färbung) der Blasendecke erfolgen. Eine Stanzbiopsie ist somit nicht unbedingt erforderlich. $\mathrm{Da}$ in den Effloreszenzen einer Id-Re-

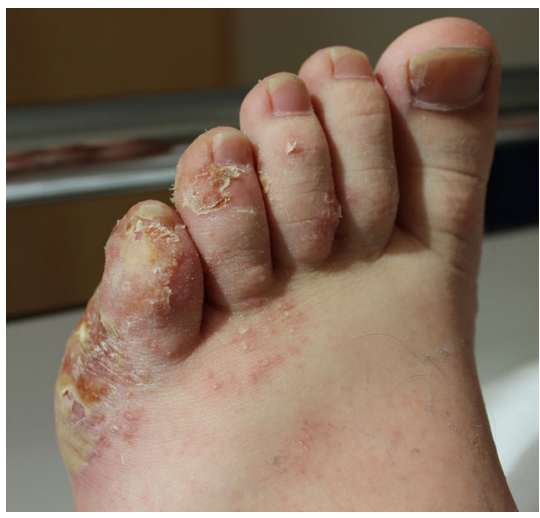

Abb. 5 - Eintrocknen der Blasen am Fuß, 3 Tage nach Therapiebeginn

aktion Pilzelemente definitionsgemäß nicht nachweisbar sein sollten, kann aus der histopathologischen Begutachtung dieser Läsionen meist nicht auf eine ursächliche Mykose geschlossen werden.

\section{》) Das Nativpräparat ist eine rasche und einfach durchzuführende Methode}

Der Intrakutantest mit Trichophytin, wie er in Form eines Stempeltests mit 7 Recall-Antigenen (Multitest Merieux ${ }^{\circledR}$, Immignost $^{\circledR}$ ) zur Verfügung stand, ist seit 2007 kommerziell nicht mehr erhältlich [23]. Sein diagnostischer Wert ist auch begrenzt, da er - in Analogie zum Tuberkulintest - nur eine stattgehabte Auseinandersetzung mit Dermatophytenallergenen signalisiert und nur eine Titration Akuität anzeigen könnte. Eine fehlende zelluläre Immunität gegenüber Trichophytin könnte jedoch bedeutsam sein bei Patienten mit chronischen, wenig entzündlichen Dermatophytosen, 
da sie auf eine gestörte Immunantwort verweisen könnte.

\section{Pathogenetische Konzepte}

Dermatophyten als häufigste Auslöser superfizieller Mykosen sind in der Lage, keratinisierte Strukturen wie Stratum corneum, Haare, Nägel unter dem klinischen Bild einer Tinea zu infizieren. Derzeit sind etwa 40 differente Spezies bekannt, die in 3 Genera klassifiziert werden: Trichophyton (T.), Microsporum (M.) und Epidermophyton (E.), während die seltener den Menschen infizierenden zoophilen und geophilen Spezies den Genera Arthroderma, Lophophyton und Nannizzia zugeordnet werden [24]. Nach der Adhärenz/Inokulation infektionsfähiger Pilzelemente an/in das Zielgewebe kommt es zum Durchwachsen der keratinhaltigen Strukturen unter Ausbildung von Myzel, wobei Keratinasen und weitere Enzyme bedeutsam sind [25-31]. Tiermodelle und Analysen von Proteinen, die aus dem infizierten Nagelbett von Patienten mit Onychomykose isoliert wurden, zeigen, dass Endoproteasen aus der Subtilisin-Familie (Sub1, Sub2, Sub7 und insbesondere Sub6) sowie die Dipeptidylpeptidase DppV die bedeutsamsten Proteasen während der Infektion darstellen $[29,30]$. Diese Proteasen scheinen aber auch wichtige Zielstrukturen für die Auslösung der Immunantwort des Wirtes zu sein. So wurden Sub6 und DppV in T. rubrum als die Hauptallergene von Dermatophyten (Tri r2 bzw. Tri r4) identifiziert [26]. Insbesondere Tri $r 2$ kann eine $\mathrm{Th}_{1}$ mediierte Immunantwort vom verzögerten Typ (DTH) induzieren.

Ein Dermatophyt provoziert eine intensivere und stärker inflammatorische Reaktion bei einem Wirt, an den er im Gegensatz zu seinem natürlichen Wirt nicht adaptiert ist. Obwohl die Zytokinaktivierung (insbesondere TNFa, IL-1b, IL-8, IL-16) in entzündlichen und nichtentzündlichen Tineaformen vergleichbar ist, unterscheidet sich insbesondere die zelluläre Immunantwort $[26,27]$. Zoophile und geophile Dermatophyten induzieren eine DTH, die beim Menschen als „Fehlwirt“ gewöhnlich in starker Inflammation, aber auch in einer spontanen Heilung und mögli- chem Schutz vor Reinfektion resultiert. Im Gegensatz dazu gehen anthropophile Spezies (wie T. rubrum, T. interdigitale und T. tonsurans) im Allgemeinen mit weniger entzündlichen, dabei mehr chronischen und persistierenden Infektionsverläufen einher. Diese Infektionen sind gekennzeichnet durch eine schwache spezifische DTH, erhöhte IgEund $\mathrm{IgG}_{4}$-Spiegel sowie durch eine IgEvermittelte Überempfindlichkeit vom Soforttyp mit einer $\mathrm{Th}_{2}$-mediierten Immunantwort [26, 27, 31].

Eine differenzielle Sekretion von Proteinen des Erregers [28-31] kann daher neben genetischen Faktoren [32] und einer individuellen Disposition des Erkrankten für die variable Induktion von Entzündung und damit auch die Ausbildung von Mykiden als begleitender hypererger Reaktion verantwortlich sein. Da das an den Menschen gut angepasste T. rubrum im Krankengut heute einen Anteil von 80-90 \% der Dermatophytenisolate erreicht [8], könnte dies auch die aktuell geringere Zahl von Mykidreaktionen erklären.

\section{》) T. rubrum erreicht im Krankengut einen Anteil von $80-90 \%$ der Dermatophytenisolate}

Ferner wirken Zellwand-Mannane und Mykotoxine wie Xanthomegnin, das insbesondere von T. rubrum gebildet wird, immunmodulatorisch und immunsuppressiv. Auch diese Verbindungen könnten auf die geringere Mykidhäufigkeit bei Infektionen durch T. rubrum einen Einfluss haben [27, 31, 33].

\section{Therapie}

Zur Therapie eines Dermatophytids ist die alleinige Gabe eines topischen Glukokortikoids meist nicht ausreichend. Wichtig ist die Beseitigung der Mykose, womit das Dermatophytid abheilt. Da es sich meist um hochentzündliche, tief abszedierende Tineaformen (Kerion Celsi, Tinea barbae) oder um Mykosen der Leistenhaut handelt, ist zumeist eine systemische antimykotische Therapie mit einem gegen Dermatophyten wirksamen Antimykotikum erforderlich. Daneben ist eine schnelle Linderung der Entzündung und des Juckreizes erwünscht. In ausgedehnten Fällen kann dies die orale Gabe eines Glukokortikoids erforderlich machen. Durch die topische Anwendung eines Antimykotikums mit dem Glukokortikoid kann neben den Entzündungssymptomen wie Rötung und Nässen insbesondere der quälende Juckreiz schnell gelindert werden. Beide Wirkstoffe zusammen wirken synergistisch hinsichtlich einer raschen Rückbildung von Erythem, Ödem, Juckreiz, Infiltrat und einer nachhaltigen antimykotischen Wirkung [34, 35]. So konnten Täuber et al. [36] zeigen, dass durch den vasokonstriktorischen Effekt der Kortikoide ein schneller Abtransport des Lokalantimykotikums durch das Gefäßsystem verhindert wird, wodurch die lokale Antimykotikumkonzentration in der Haut in der kombinierten Anwendung um das 2- bis 3-Fache gegenüber der Monosubstanz gesteigert werden konnte. Ferner weisen Breitspektrumantimykotika aus der Gruppe der Azole wie Miconazol auch eine Wirksamkeit gegenüber einer Superinfektion mit grampositiven Bakterien wie Staphylococcus aureus auf [37], wie sie gerade bei der dyshidrosiformen Tinea pedis häufig beobachtet wird (• Abb. 1). Da es sich bei den Veränderungen eines Dermatophytids um eine hypererge, "sterile“ Reaktion handelt, kann bei diesen Läsionen ein topisches Steroid mit hoher antiphlogistischer Wirkung und geringer Atrophogenität als Monopräparat zur Anwendung kommen.

》W Wichtig ist die Beseitigung
der Mykose, womit das
Dermatophytid abheilt

Dem dyshidrosiformen Mykid scheint im klinischen Alltag die größte Bedeutung zuzukommen. Dyshidrosiforme Veränderungen im Bereich der Hände haben eine breite Differenzialdiagnose und sind therapeutisch oft schwer zugänglich. Zwar findet sich häufig eine Hyperhidrose, aufgrund der histopathologischen Veränderungen sind diese 
Veränderungen aber den Dermatitisund Ekzemerkrankungen zuzuordnen. Eine Reihe von Ursachen ist möglich und bestimmt das therapeutische Vorgehen.

Hierzu zählen [1, 12-15]:

- eine Dyshidrose im Rahmen einer atopischen Diathese,

- eine Kontaktallergie (z. B. im Bereich der Schuhe durch Lederfarbstoffe oder Kleber mit Streureaktionen im Bereich der Hände)

- ein hämatogenes Kontaktekzem auf die systemische Aufnahme von Allergenen wie Nickel oder Perubalsam

- Arzneimittelreaktionen sowie

- psychogene Ursachen bei emotionalen Stressreaktionen.

Eine günstig zu beeinflussende Ursache sind dyshidrosiforme Mykide bei Tinea pedis/pedum. Dyshidrosiforme Veränderungen im Bereich der Hände sollten daher Veranlassung geben, nach einer Fußmykose zu schauen, da die antimykotische Therapie zu einer dauerhaften Abheilung der dyshidrosiformen Veränderungen führt. Die überwiegende Anzahl dieser Patienten weist eine Infektion mit dem Erreger T. mentagrophytes auf, der meist zu starken Entzündungen mit teilweise bullösen Läsionen der Fußsohlen führt. Bei diesem Erreger können dyshidrosiforme Mykide im Bereich der Hände in etwa einem Drittel der Fälle beobachtet werden [14].

Da die Inzidenz der Tinea capitis in Mitteleuropa ansteigt und sich darüber hinaus das Erregerspektrum wandelt [7, 8], ist auch hier vermehrt mit dem Auftreten von Dermatophytiden zu rechnen. Neben dem Lichen trichophyticus umfassen die Tinea-capitis-assoziierten Mykide ein breites klinisches Spektrum und können vor und nach Einleitung einer systemischen antimykotischen Therapie auftreten. Gerade ein Auftreten unter Therapie macht die Abgrenzung von einer Arzneireaktion bedeutsam. Andererseits könnte eine $\mathrm{zu}$ rasch beendete systemische antimykotische Therapie zu einer ausbleibenden Abheilung der Dermatophytose und der assoziierten Id-Reaktion führen.

Wenn ein Mykid erst nach Beginn einer systemischen antimykotischen Therapie auftritt, wurde das von manchen
Autoren als „Overtreatment-Phänomen“ bezeichnet [1, 34, 38]. Solche entzündlichen Aufflammreaktionen werden v. a. bei hochentzündlichen Tineaformen durch zoophile Dermatophyten beobachtet und finden sich besonders unter Therapie mit den neuen, hochwirksamen Antimykotika wie Fluconazol, Itraconazol und Terbinafin. Sie beruhen möglicherweise auf einer verstärkten Antigenfreisetzung des rasch absterbenden Pilzes. Nikkels et al. [39] beschrieben über einen Beobachtungszeitraum von 10 Jahren 5 Personen, die ein „flareup“ der Entzündung nach Beginn einer oralen antimykotischen Therapie zeigten. Es handelte sich um 4 Landwirte und 1 Tierarzt, die an entzündlichen Dermatophytosen, ausgelöst vornehmlich durch T. verrucosum, litten. Die Verschlechterung begann 12-24 h nach Einnahme der oralen Antimykotika. Milde Systemzeichen mit mäßigem Fieber und Schwächegefühl traten bei 2 Patienten auf. Itraconazol $400 \mathrm{mg} / \mathrm{Tag}$ wurde in 4 Fällen, Terbinafin $250 \mathrm{mg} / \mathrm{Tag}$ in 1 Fall verabreicht. Die systemische antimykotische Therapie wurde bei allen Patienten beendet, orale (Patienten mit Systemzeichen) und topische Steroide wurden verabreicht. Zwei Tage nach Kortikoidgabe wurden niedere Dosen von Itraconazol (100 mg/Tag) und Terbinafin (125 mg/Tag) wieder eingeführt. Alle Veränderungen heilten nach 4 bis 5 Wochen ab.

Das Overtreatment-Syndrom wurde zunächst als eine Form der JarischHerxheimer-Reaktion (JHR) diskutiert, wobei aber nicht alle Kriterien erfüllt waren. Die JHR ist charakterisiert durch eine Exazerbation der bestehenden Hautveränderungen mit rasch einsetzenden Systemzeichen innerhalb von 4-8 h nach Therapiebeginn. Eine starke Freisetzung von bakteriellen Bestandteilen/ Endotoxinen führt zu einem Zytokinsturm (Erhöhung von TNF- $\alpha$, IL-6 und IL-8). Das Overtreatment-Syndrom zeigt gewöhnlich eine 12- bis 24-stündige Latenz zwischen Medikamenteneinnahme und verstärkter Entzündungsreaktion, vergleichbar einem allergischen Kontaktekzem mit ausgeprägter Sensibilisierung und verstärkter Reaktion bei wiederholter Allergenexposition. Das klinische
Spektrum ist breit. Es kann als lokale Exazerbation am Infektionsort auftreten, als Verschlechterung eines ggf. zuvor bestehenden Mykids oder als generalisierte exanthematische Reaktion mit gestörtem Allgemeinbefinden. In letzterem Fall ist eine Abgrenzung von einer Arzneireaktion schwierig. Insbesondere das Auftreten generalisierter pustulöser Dermatophytide $[19,20]$ macht eine Abgrenzung von einer akuten generalisierten exanthematischen Pustulose (AGEP) erforderlich. Gerade das bei Dermatophytosen hochwirksame Allylamin Terbinafin zählt bei systemischer Verabreichung zu den Medikamenten mit einem hohen Risiko für eine AGEP [39].

Da Nikkels et al. [38] nur 5 Fälle in einem 10-jährigen Zeitraum beschreiben konnten, könnten Aufflammreaktionen durch die Gabe von Antimykotika selten oder einfach nur wenig beschrieben sein. Es gibt daher auch nur wenige Angaben, welche Maßnahmen zu ergreifen sind. Besonders beim Auftreten von Allgemeinsymptomen ist es wichtig, die systemische Gabe des Antimykotikums zu beenden, da eine Arzneireaktion nicht ausgeschlossen werden kann. Da aber die Mykose zu diesem Zeitpunkt meist nicht ausreichend behandelt ist, sollte die antimykotische Therapie fortgesetzt werden, entweder mit einer niederen Dosis oder bevorzugt mit einer Änderung der Substanzklasse (Terbinafin gegen Azol bzw. umgekehrt). Neben einer ggf. kurzfristigen Gabe oraler Steroide sind topische Kortikoide in Kombination mit Antimykotika hilfreich, um die Entzündung zu reduzieren und die Pilzinfektion zu behandeln.

\section{Fazit für die Praxis}

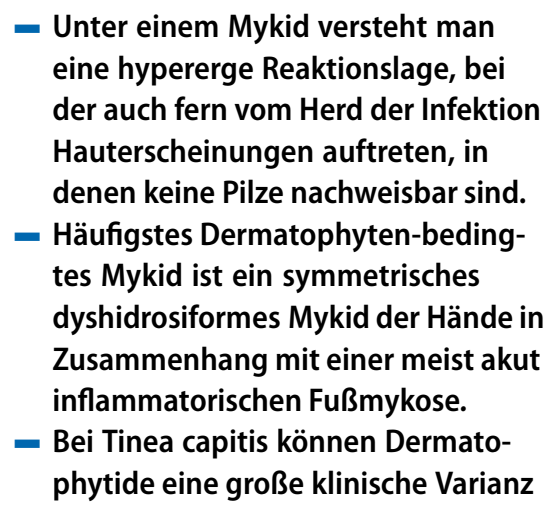




\section{aufweisen und treten nicht selten erst unter der erforderlichen systemi- schen antimykotischen Therapie auf. Sie müssen von einer Arzneireaktion abgegrenzt werden. \\ - Bei Tinea capitis ist meist eine sys- temische antimykotische Therapie, bei stark entzündlicher Komponen- te sind ggf. auch kurzfristig orale Glukokortikoide erforderlich. \\ - Die Lokaltherapie in Form einer Kombination eines Antimykotikums mit einem Glukokortikosteroid zielt auf eine schnelle Linderung der entzündlichen Veränderungen und des Juckreizes. \\ - Da es sich bei den Veränderungen im Bereich der Hände um eine hy- pererge, "sterile“ Reaktion handelt, kann auch ein topisches Glukokorti- koidmonopräparat zur Anwendung kommen.}

\section{Korrespondenzadresse}

\section{Prof. Dr. P. Mayser}

Hofmannstr. 11, 35444 Biebertal, Deutschland peter.mayser@derma.med.uni-giessen.de

\section{Einhaltung ethischer Richtlinien}

Interessenkonflikt. P. Mayser war als Berater und/ oder Redner für folgende Firmen tätig: Almirall-Hermal, Medupdate, Novartis, OmniaMed, Pierre Fabre.

Dieser Beitrag beinhaltet keine vom Autor durchgeführten Studien an Menschen oder Tieren.

Open Access. Dieser Artikel wird unter der Creative Commons Namensnennung 4.0 International Lizenz (http://creativecommons.org/licenses/by/4.0/deed. de) veröffentlicht, welche die Nutzung, Vervielfältigung, Bearbeitung, Verbreitung und Wiedergabe in jeglichem Medium und Format erlaubt, sofern Sie den/die ursprünglichen Autor(en) und die Quelle ordnungsgemäß nennen, einen Link zur Creative Commons Lizenz beifügen und angeben, ob Änderungen vorgenommen wurden.

\section{Literatur}

1. Ilkit M, Durdu M, Karakaş M (2012) Cutaneous id reactions: a comprehensive review of clinical manifestations, epidemiology, etiology, and management. Crit Rev Microbiol 38:191-202

2. Jadassohn J (1918) Über die Trichophytien. Klin Wochenschr 21:489-494

3. Bloch B (1928) Allgemeine und experimentelle Biologie der Dermatomykosen und Trichophytide. In: Jadassohn J (Hrsg) Handbuch der Haut- und
Geschlechtskrankheiten, Bd. 11. Springer, Berlin, S 300-376(564-606)

4. Seebacher C (1994) Heutiger Stand des MykidKonzeptes. Akt Dermatol 20:406-410

5. Bryld LE, Agner T, Mennè T (2003) Relation between Vesicular eruptions on the Hands and tinea pedis, atopic dermatitis and nickel allergy. Acta Derm Venereol 83:186-188

6. Burzykowski T,MolenberghsG, AbeckDetal (2003) High prevalence of foot diseases in Europe: results of the Achilles Project. Mycoses 46:496-505

7. RuhnkeM,Groll AH, Mayser Petal (2015) Estimated burden of fungal infections in Germany. Mycoses 58(Suppl 5):22-28

8. Mayser P (2012) Mykosen. In: Plewig G, Landthaler M, Burgdorf WHC, Hertl M, Ruzicka T (Hrsg) Braun-Falco's Dermatologie, Venerologie und Allergologie, 6. Aufl. Springer, Berlin, S 252-257

9. Grappel SF, Bishop CT, Blank F (1974) Immunology of dermatophytes and dermatophytosis. Bacterio Rev 38:222-250

10. Elmofty AM, Nada MM (1965) Erythema anulare centrifugum as a dermatophytid. Hautarzt 16:123-125

11. Gianni C, Betti R, Crosti C (1996) Psoriasiform idreaction in tinea corporis. Mycoses 39:307-308

12. Mayser P, Latussek E (2009) Ausgeprägtes dyshidrosiformes Mykid als Komplikation einer Tinea pedis. Akt Dermatol 35:1-4

13. Romano C, Rubegni P, Ghilardi A, Fimiani M (2006) A case of bullous tinea pedis with dermatophytid reaction caused by Trichophyton violaceum. Mycoses 49:249-250

14. Veien NK, Hattel T, Laurberg G (1994) Plantar Trichophyton rubrum infections may cause dermatophytids on the hands. Acta Derm Venereol 74:403-404

15. Kaaman T (1981) Cell-mediated reactivity in dermatophytosis: differences in skin responses to purified trichophytin in tinea pedis and tinea cruris Acta Derm Venereol 61:119-123

16. Dostrovsky A, Kallner G, Raubitschek F, Sagher F (1955) Tinea capitis: an epidemiologic, therapeutic and laboratory investigation of 6390 cases. J Invest Dermatol 24:195-200

17. Cheng N, Rucker Wright D, Cohen BA (2011) Dermatophytid in tinea capitis: rarely reported common phenomenon with clinical implications. Pediatrics 128:e453-e457

18. Topaloğlu Demir F, Karadag AS (2015) Are dermatophytid reactions in patients with Kerion Celsi much more common than previously thought? A prospective study. Pediatr Dermatol 32:635-640

19. Liu ZH, Shen H, Xu AE (2011) Severe kerion with dermatophytid reaction presenting with diffuse erythema and pustules. Mycoses 54:e650-e652

20. Ronjat L, Ferneiny M, Hadj-Rabia S et al (2015) Generalized exanthematous pustular dermatophytid, a rare clinical presentation of dermatophytid reaction. Ann Dermatol Venereol 142:270-275

21. Zaraa I, Trojjet S, El Guellali N et al (2012) Childhood erythema nodosum associated with kerion celsi: a case report and review of literature. Pediatr Dermatol 29:479-482

22. Castriota M, Ricci F, Paradisi A et al (2013) Erythema nodosum induced by kerion celsi of the scalp in a child: a case report and mini-review of literature. Mycoses 56:200-203

23. Bekanntmachungen des Paul-Ehrlich-Instituts BundesanzeigerNr. 58; 3/2007

24. de Hoog GS, DukikK, Monod Metal (2016) Toward a novel multilocus phylogenetic taxonomy for the dermatophytes. Mycopathologia.doi:10.1007/ s11046-016-0073-9

25. Brasch J (2010) Pathogenesis of tinea. J Dtsch Dermatol Ges 8:780-786

26. Woodfolk JA (2005) Allergy and dermatophytes. Clin Microbiol Rev 18:30-43

27. Almeida SR (2008) Immunology of dermatophytosis. Mycopathologia 166:277-283

28. Burmester A, Shelest E, Glöckner G et al (2011) Comparative and functional genomics provide insights into the pathogenicity of dermatophytic fungi. Genome Biol 12:R7

29. Staib P, Zaugg C, Mignon B et al (2010) Differential gene expression in the pathogenic dermatophyte Arthroderma benhamiae in vitro versus infection. Microbiology 156:884-895

30. Méhul B, Gu Z, Jomard A et al (2015) Sub6 (Tri $r$ 2), an onychomycosis marker revealed by proteomics analysis of Trichophyton rubrum secreted proteins in patient nail samples. J Invest Dermatol 2016(136):331-333

31. Vermout S, Tabart J, Baldo A et al (2008) Pathogenesis of dermatophytosis. Mycopathologia 166:267-275

32. García-Romero MT, Arenas R (2015) New insights into genes, immunity, and the occurrence of dermatophytosis. J Invest Dermatol 135:655-657

33. Gupta AK, Ahmad I, Borst I, Summerbell RC (2000) Detection of xanthomegnin in epidermal materials infected with Trichophyton rubrum. J Invest Dermatol 115:901-905

34. Mayser $P$ (2016) Versorgung von Hauterkrankungen: Stellenwert und Einsatz von Glukokortikosteroiden in fixer Kombination mit Antimykotika. Hautarzt 67:732-738

35. Schaller M, Friedrich M, Papini Metal (2016) Topical antifungal-corticosteroid combination therapy for the treatment of superficial mycoses: conclusions of an expert panel meeting. Mycoses 59:365-373

36. Tauber U, Rzadkiewicz M (1979) Bioverfügbarkeit von Isoconazol in der Haut. Mykosen 22:201-216

37. Nenoff $P$, Koch $D$, Krüger $C$ et al. Efficacy of miconazole against gram positive - incl. MRSA, MSSA and FRSA, gram negative, aerobe and anaerobe bacteria relevant in skin diseases - an invitro investigation. Mycoses (submitted)

38. Nikkels AF, Nikkels-Tassoudji N, Piérard GE (2006) Oral antifungal-exacerbated inflammatory flareup reactions of dermatomycosis: case reports and review of the literature. Am J Clin Dermatol 7:327-331

39. Mayser $P$ (2016) Terbinafine: drug-induced lupus erythematosus and triggering of psoriatic skin lesions. Hautarzt 67:724-731 